\title{
Cutaneotrichosporon (Cryptococcus) cyanovorans, a basidiomycetous yeast, described for the first time from the airways of cystic fibrosis patients
}

Tjomme van der Bruggena, Anna Koleckab ${ }^{\text {, Bart Theelen }}{ }^{b}$, Johanna M. Kwakkel-van Erpa ${ }^{a}$ Bert Arets ${ }^{a}$, and Teun Boekhout ${ }^{b}$ a University Medical Center Utrecht, The Netherlands, b Westerdijk Fungal Biodiversity Institute, Utrecht, The Netherlands

\section{Abstract}

Cystic fibrosis (CF) patients are colonized by a multitude of bacteria and fungi. In our institute, respiratory specimens from a large part of the Dutch CF population is frequently cultured. Here, we report the repeated isolation of Cutaneotrichosporon (Cryptococcous) cyanovorans from the airways of two CF patients. C. cyanovorans was described in 2012 as a basidiomycetous yeast isolated from South African soil, contaminated with cyanide. ${ }^{1}$

\section{Methods}

Identification methods consisted of matrix assisted laser desorption/ionization time-of-flight mass spectrometry (MALDITOF-MS) using the MALDI biotyper (Bruker, Germany) and internal transcribed spacer (ITS) and ribosomal large subunit (LSU) sequencing. Antifungal susceptibility was tested with a microdilution method (Yeastone, Trek Diagnostic Systems, UK).

Table 1. In vitro susceptibility of $C$. cyanovorans as determined by microdilution

\begin{tabular}{|c|c|c|}
\hline & $\begin{array}{c}\text { C. cyanovorans } \\
\text { (strain K314) } \\
\text { Patient 1 }\end{array}$ & $\begin{array}{c}\text { C. cyanovorans } \\
\text { (strain G2100) } \\
\text { Patient 2 }\end{array}$ \\
\hline Antifungal agent & \multicolumn{2}{|c|}{$\mathrm{MIC}(\mu \mathrm{g} / \mathrm{ml})$} \\
\hline Fluconazole & 8 & 8 \\
\hline Itraconazole & 0.25 & 0.5 \\
\hline Voriconazol & 0.25 & 0.5 \\
\hline Posaconazole & 0.5 & 1 \\
\hline Anidulafungin & $>8$ & $>8$ \\
\hline Micafungin & $>8$ & $>8$ \\
\hline Caspofungin & $>8$ & $>84$ \\
\hline Flucytosine & $>64$ & 1 \\
\hline Amphotericin B & 1 & \\
\hline
\end{tabular}

Figure 1. MALDI-TOF MS based dendrogram

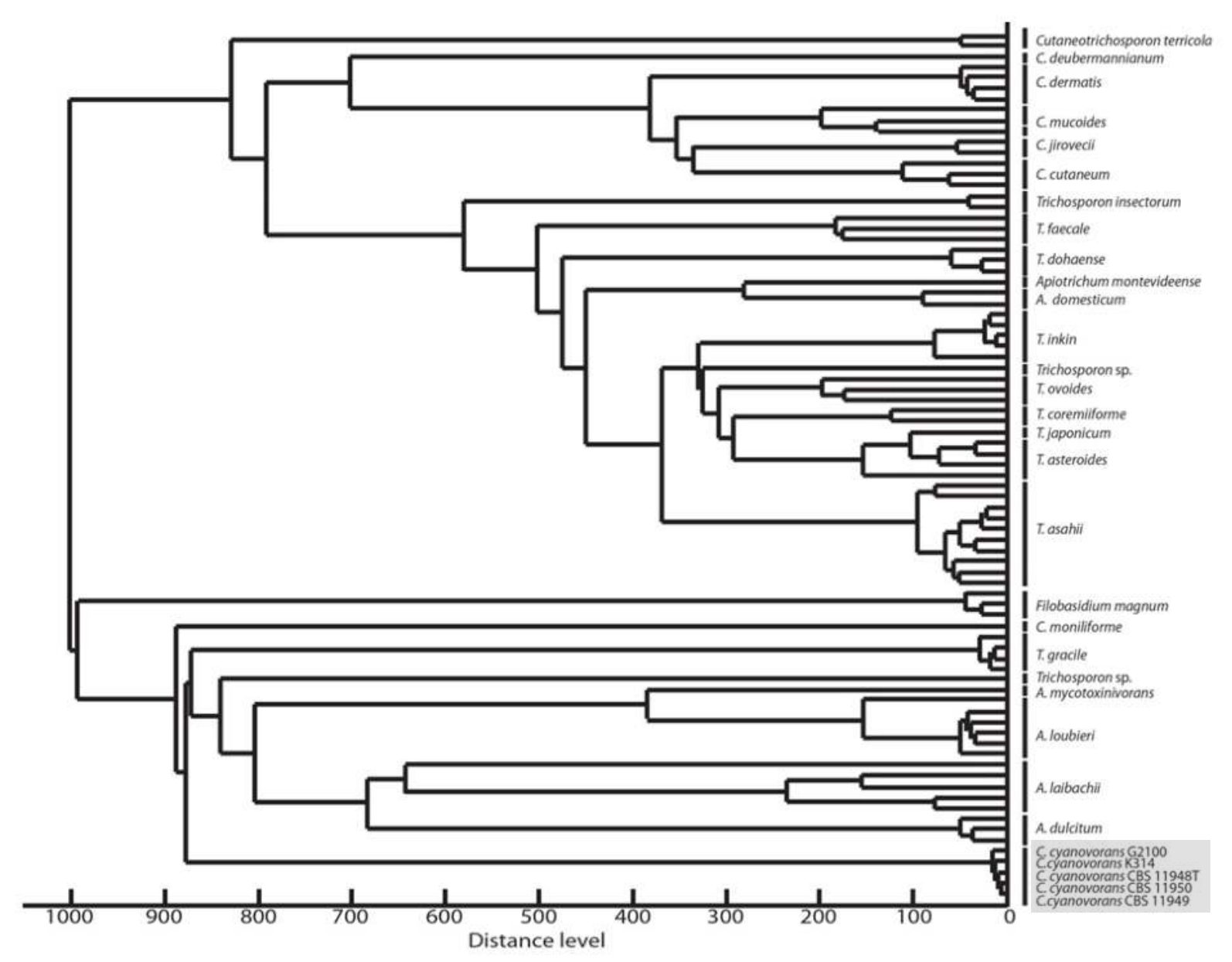

Patient 1 This is a 37 year old female CF patient (homozygous $\Delta \mathrm{F} 508$ ), who received bilateral lung transplantation in 2017. Several sputum samples were positive from 2014-2017 for a yeast that could not be identified with MALDI-TOF MS. The yeast grew on a malt agar, containing chloramphenicol, colistin and vancomycin, after $1-2$ days of culture at $37^{\circ} \mathrm{C}$ in ambient air. Two of these isolates were identified as $C$. cyanovorans by ITS and LSU sequence analysis showing full agreement with sequences of the South African environmental strains (CBS $11948 / 11949 / 11950)$. The antifungal susceptibility is shown in table 1 . The patient received voriconazole after transplantation. She has never been in South Africa.

Patient 2 This is a 20 year old female with CF (homozygous $\Delta$ F508). From 2012 to 2015, sputum and throat swab cultures were frequently positive for a difficult to identify yeast by MALDITOF MS. The yeast grew on malt agar after two days at $37^{\circ} \mathrm{C}$. Two isolates were shown to be $C$. cyanovorans based on ITS and LSU sequencing. The antifungal susceptibility profile was comparable to the isolate of patient 1 (Table 1).

A dendrogram based on mass spectra of the South African strains and clinical isolates shows clustering of the patient and reference strains and clear separation from other yeasts (Figure 1). The patient was not treated with antifungals. She has never travelled to South Africa.

\section{Discussion}

C. cyanovorans was originally isolated from cyanide contaminated soil in South Africa. Phylogenetic analysis showed that the clinical isolates are closely related to the South African strains. However, both patients have never been in South Africa. C. cyanovorans can grow in the presence of cyanide in vitro. In general, cyanide is present in the lungs of many CF patients, suggesting the possibility of an advantageous pulmonary environment in CF for $C$. cyanovorans. However, we were not able to perform a measurement of cyanide levels in our patients.

\section{Conclusion}

The presence of $C$. cyanovorans in respiratory specimen of CF patients is a novel finding. Further study is needed to determine whether more positive CF patients can be identified. If so, a possible relation with cyanide in the CF lung and the clinical significance can be investigated. 